
\title{
28 Research Suare \\ Effects of SGLT2 Inhibitor on Left Ventricular Function: A Meta-analysis
}

\section{OU Zhenfei ( $\nabla$ 635070418@qq.com )}

Affiliated hospital of qindao university https://orcid.org/0000-0002-1727-8717

\section{Chen Tong}

The Affiliated Hospital of Qingdao University

\section{Original investigation}

Keywords: SGLT-2 inhibitors, Type 2 diabetes, Left ventricular remodeling

Posted Date: November 5th, 2020

DOl: https://doi.org/10.21203/rs.3.rs-101454/v1

License: (c) (i) This work is licensed under a Creative Commons Attribution 4.0 International License. Read Full License 


\section{Abstract}

Background: Although a variety of research have significant protective action on the cardiovascular system when use of SGLT2 inhibitor, it is still unclear how can improve ventricular remodeling and fundamentally Reduce the mortality of cardiovascular. The purpose of this meta-analysis is to explore the efficacy of sodium glucose cotransporter type 2 (SGLT-2) inhibitors in improving left ventricular (LV) remodeling in patients with type 2 diabetes (T2DM).

Methods: We searched articles published before September 30, 2020, regardless of language, in 4 electronic databases: PubMed, EMBASE, Cochrane Library and Web of Science. We included randomized controlled trials in this meta-analysis. The differences in the mean changes in left ventricular echocardiographic parameters between the treatment group and control group were evaluated.

Results: Combined outcome indicators showed that SGLT2 inhibitors, LAVI (WMD囚-6.29ه95هCI

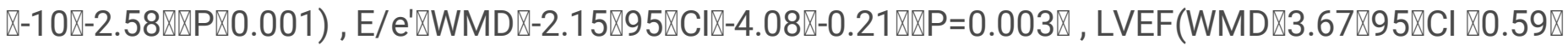

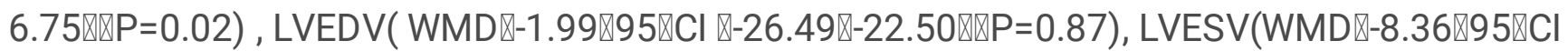
$\bigotimes-17.36 \rrbracket-0.65 \otimes \mathbb{P}=0.07)$.

Conclusions: SGLT2 inhibitors had a favorable effect on left ventricular systolic and diastolic function in patients with T2DM.

\section{Background}

Currently, there are 382 million people with diabetes in the world, which will increase to 592 million in $2035,90 \%$ of which are type 2 diabetes patients [1],Compared with non-diabetic patients, diabetic patients have twice the cardiovascular risk and are more prone to left ventricular dysfunction and heart failure [3-4], But intensive hypoglycemia does not reduce cardiovascular risk [5]. Until the advent of SGLT2 inhibitors, breaking this deadlock, Results from a large cardiovascular trial in patients with type 2 diabetes suggest that sodium-glucose co-transporter 2 (SGLT2) inhibitors may improve cardiovascular outcomes, particularly by reducing the risk of hospitalization for heart failure. [6-9]

The current pharmacological treatments for heart failure include diuretics, angiotensin-converting enzyme inhibitors (ACEI)/angiotensin II receptor antagonists (ARBs), beta-blockers, sacubatril valsartan, aldosterone receptor antagonists, and digoxin. [10]区SGLT2 inhibitors are designed to lower blood glucose levels by specifically inhibiting glucose reabsorption from the proximal renal tubules, and recent studies suggest cardiac benefits. Several clinical studies have been conducted on the effects of SGLT2 inhibitors on ventricular structure and function. Although many hypotheses have been proposed, the mechanism by which it reduces hospitalization in patients with heart failure remains uncertain, and the impact on cardiac function is controversial. Left ventricular mass (LVM) is an independent predictor of cardiovascular events such as myocardial infarction, hospitalization for heart failure and mortality. [1112] We performed a meta-analysis of randomized controlled trials of cardiac function to assess the effect 
of SGLT2 inhibitors on cardiac remodeling in patients with type 2 diabetes mellitus. Analyze the role of this drug in cardiac remodeling and provide data to support the drug mechanism.

\section{Methods}

\section{Eligibility criteria}

We included randomized controlled trials with parallel group or crossover designs in this meta-analysis, Study participants were type 2 diabetic patients, The outcome of the included studies must contain the following 6 cardiac function and structure measures: LVEF, LV end-diastolic diameter (LVEDD), LV endsystolic diameter (LVESD), LV end-diastolic volume (LVEDV), LV end-systolic volume (LVESV), LV mass index (LVMI) and mitral inflow E velocity to tissue Doppler e'ratio (E/e').

\section{Information sources and search strategy}

We searched articles published before September 30, 2020, regardless of language, in 4 electronic databases: PubMed, EMBASE, Cochrane Library and Web of Science. The articles were selected by manual screening

The following terms were used in the search: ventricular remodeling OR cardiac reverse remodeling OR CRR OR cardiac remodeling OR left ventricular remodeling OR left ventricular dysfunction OR LVD OR ejection fraction OR EF OR left ventricular ejection fraction OR LVEF OR end-diastolic volume OR EDV OR end-diastolic dimension OR EDD OR end-systolic volume OR ESV OR end-systolic dimension OR ESD OR LVEDD OR left ventricular end-diastolic dimension OR LVEDV OR left ventricular end-diastolic volume OR LVESD OR left ventricular end-systolic dimension OR LVESV OR left ventricular end-systolic volume OR left ventricular diameter OR left ventricular volume OR left ventricular mass index OR LVMI OR left atrial volume OR LAV OR left atrial volume index OR LAVI) AND (canagliflozin OR dapagliflozin OR empagliflozin OR ertugliflozin OR ipragliflozin OR luseogliflozin OR tofogliflozin)

\section{Study selection}

Two methodologically trained independent reviewers screened titles and abstracts to determine whether they met the eligibility criteria. The reviewers read the full text and extracted relevant data after consensus was reached. Any differences were resolved through discussion and arbitration, if necessary, by a third reviewer. The reasons for inclusion or exclusion are recorded in detail. Case reports, letters and minutes of meetings were excluded.

\section{Definition of outcomes}

The outcome of this meta-analysis was the difference in the mean change in echocardiographic parameters between the treatment group and control group. The echocardiographic parameters included LVEF, LVEDV, LVESV, LVMI and E/e'. 


\section{Statistical analysis}

We calculated weighted mean differences (WMDs) with 95\% confidence intervals (Cls) to assess effect size for continuous variables including LVEF, LVEDV, LVESV, LVMI and E/e'. In the meta-analysis, we used a random effects model to combine estimators. We also considered a fixed effect model additionally for exploration of the discrepancy in results. The $\mathrm{I}^{2}$ statistic, $\mathrm{T}^{2}$ statistic, and Cochran's $\mathrm{Q}$ test were used to assess statistical heterogeneity among the studies. All statistical analyses were performed using $\mathrm{R}$ version 3.1.0 ( $R$ Foundation for Statistical Computing, Vienna, Austria). $P$ values of $<0.05$ was regarded as statistically significant for treatment effects and test for heterogeneity, respectively.

\section{Results}

\section{Difference in mean change in LEVF}

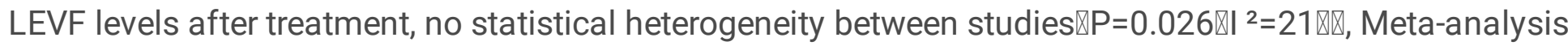
using a fixed effects model (Figure 2). Meta-analysis showed a statistically significant difference in post-

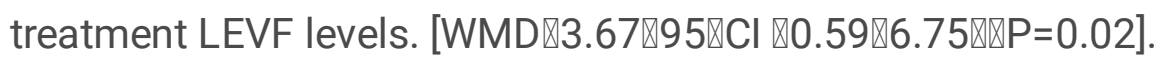

\section{Difference in mean change in LAVI}

Post-treatment LAVI levels, no statistical heterogeneity between studies. $\mathrm{QP}=\left.0.46 \mathrm{~W}\right|^{2}=0 \mathrm{Q} \mathrm{Q}$, Meta-analysis using fixed effects model, (Figure 3). Meta-analysis showed a statistically significant difference in post-

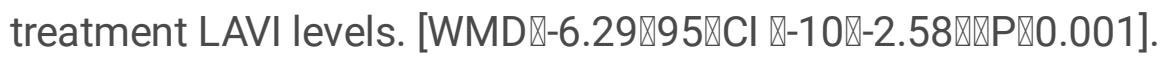

\section{Difference in mean change in $E / E^{\prime}$}

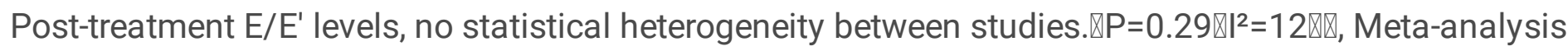
was performed using a fixed-effects model (Figure 4). Meta-analysis showed a statistically significant

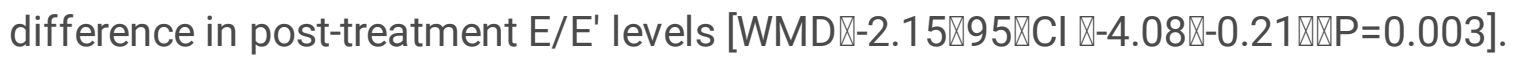

\section{Difference in mean change in LVEDV}

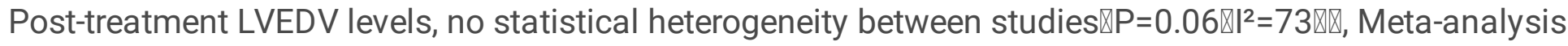
was performed using a stochastic model (Figure 5). Meta-analysis showed that there was no statistically significant difference in the comparison of LVEDV levels after treatment [WMD囚-1.99ه95खCl $\bigotimes-26.49 \rrbracket-22.50 \otimes \otimes \mathrm{P}=0.87]$.

\section{Difference in mean change in LVESV}

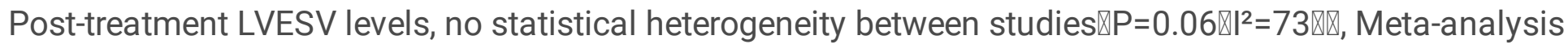
was performed using a fixed model (Figure 6). Meta-analysis showed that there was no statistically significant difference in the comparison of LVESV levels after treatment [WMD囚-8.36₫95囚CI $\bigotimes-17.36 \rrbracket-0.65 \bigotimes \bigotimes P=0.07]$. 


\section{Discussion}

SGLT2 inhibitors reduce hospitalization rates in patients with heart failure, but do not affect atherosclerosis-related events, and the primary mechanism may be related to improved left ventricular function [13]. The current pharmacological mechanisms considered are: (1) renal sodium excretion, glucose excretion, diuresis, and lowering of glomerular capillary blood pressure [14]. (2) Increase oxygen supply to the heart, improve energy metabolism, reduce myocardial fibrosis, and reduce epicardial fat deposition. (3) Improved endothelial function, reduced vascular resistance, and reduced aortic wall strength. (4) Affects neurohormonal pathways in the heart and decreases activation of the sympathetic nervous system [15-16]. The above mechanisms affect cardiac remodeling by reducing cardiac pre- and postload and improving insulin resistance. [17]

Some of the current non-randomized controlled trials have demonstrated that SGLT2 inhibitors reduce left ventricular mass index and diastolic function after 3 to 6 months of treatment in diabetic patients, including empagliflozin[18],canagliflozin[19], dapagliflozin[20], tofogliflozin[21]. However, these studies included limited populations and had short follow-up periods. It has also been suggested that in patients with type 2 diabetes, tofogliflozin does not significantly improve left ventricular systolic or diastolic function [22]. The present study suggests that empagliflozin can significantly improve cardiac remodeling in patients with type 2 diabetes, with significant reductions in E/E' and LAVI levels after treatment; however, there were no significant differences in LEVF levels, LVEDV and LVESV levels after treatment. This suggests that empagliflozin may provide short-term cardiac benefit to patients, whether it improves their long-term prognosis remains to be explored.

This study has limitations (1) The literature is less than 25 weeks long and there are few studies of longterm drug prognosis. (2) The limited number of included studies and the small number of cases in some of the studies, which included patients with type 2 diabetes with different disease severity, disease duration, and whether they were co-morbid with other diseases, may lead to some heterogeneity in the results (3) As the literature with positive results is more likely to be published, there may be systematic errors. It can be seen that more large sample, high quality RCTs are needed to further confirm the conclusions reached in this study.

In summary, empagliflozin may improve cardiac remodeling. Current studies in animals and humans with diabetes suggest that SGLT2 inhibitors can potentially improve LV mass index and diastolic function. However, the biological effects of SGLT2 inhibitors on cardiac structure and function remain uncertain and need to be supported by additional studies in RCTs.

\section{Conclusion}

Our findings support the role of SGLT2 inhibitors on preservation of cardiac function. However, the evidence is currently limited to T2MD patients. There is an urgent need to investigate the T2MD outcome 
of SGLT2 inhibitors therapy outside the T2MD setting, where the evidence is still scarce and of low quality.

\section{Declarations}

Ethics approval and consent to participate

Consent for publication

Not applicable.

\section{Availability of data and materials}

Competing interests

The authors declare that they have no competing interests.

\section{Funding}

There were no sources of funding for this research.Authors' contributions

\section{Authors' contributions}

Not applicable

\section{Acknowledgments}

We thank chen tong for statistical support and Graphical assistance in this manuscript.

\section{References}

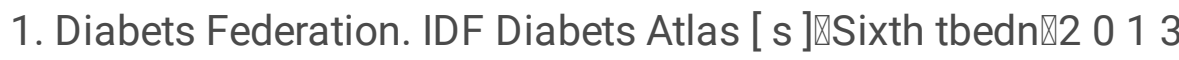

2. Di Angelantonio E, Kaptoge S, Wormser D, Willeit P, Butterworth AS, Bansal N, et al. Association of Cardiometabolic Multimorbidity with Mortality. JAMA. 2015;314(1):52-60

3. From AM, Scott CG, Chen HH (2010) The development of heart failure in patients with diabetes mellitus and pre-clinical diastolic dysfunction a population-based study. J Am Coll Cardiol 55:300305. https://doi.org/10.1016/j.jacc.2009.12.003.

4. Dawson A, Morris AD, Struthers AD (2005) The epidemiology of left ventricular hypertrophy in type 2 diabetes mellitus. Diabetologia 48:1971-1979. https://doi.org/10.1007/s00125-005-1896-y

5. Stratton IM, Adler Al, Neil HA, Matthews DR, Manley SE, Cull CA, et al. Association of glycaemia with macrovascular and microvascular complications of type 2 diabetes [UKPDS 35]: prospective observational study. BMJ Clinical Research Ed. 2000;321(7258):405-12. 
6. Neal B, Perkovic V, Mahaffey KW, et al. Canagliflozin and cardiovascular and renal events in type 2 diabetes. N Engl J Med 2017; 377: 644-570

7. Wiviott SD, Raz I, Bonaca MP, et al. Dapagliflozin and cardiovascular outcomes in type 2 diabetes. $N$ Engl J Med 2019; 380: 347-570

8. Perkovic V, Jardine MJ, Neal B, et al. Canagliflozin and renal outcomes in type 2 diabetes and nephropathy. N Engl J Med 2019;380: 2295-306.

9. Zinman B, Wanner C, Lachin JM, Fitchett D, Bluhmki E, Hantel S, Mattheus M, Devins T, Johansen OE, Woerle HJ, Broedl UC, Inzucchi SE. Empagliflozin, cardiovascular outcomes, and mortality in type 2 diabetes. N Engl J Med 2015; 373: 2117-2128.

10.

11. Abdi-Ali A, Miller RJH, Southern D, Zhang M, Mikami Y, Knudtson M, Heydari B,Howarth AG, Lydell CP, James MT, et al. LV Mass Independently Predicts Mortality and Need for Future Revascularization in Patients Undergoing Diagnostic Coronary Angiography. JACC Cardiovasc Imaging. 2018;11:423433.14.

12. Mathew J, Sleight P, Lonn E, Johnstone D, Pogue J, Yi Q, Bosch J, Sussex B, Probstfield J, Yusuf S, et al. Reduction of cardiovascular risk by regression of electrocardiographic markers of left ventricular hypertrophy by the angiotensin-converting enzyme inhibitor ramipril. Circulation. 2001;104:16151621

13. Sattar N, McLaren J, Kristensen SL, Preiss D, McMurray JJ. SGLT2 inhibition and cardiovascular events: why did EMPA-REG outcomes surprise and what were the likely mechanisms? Diabetologia 2016; 59: 1333-1339

14. Shubrook JH, Bokaie BB, Adkins SE. Empagliflozin in the treatment of type 2 diabetes: evidence to date. Drug Des Devel Ther. 2015;9:5793-803. Kalra S. Sodium glucose co-transporter-2 [SGLT2] inhibitors: a review of their basic and clinical pharmacology. Diabetes Ther. 2014;5(2):355-66

15. Chilton R, Tikkanen I, Cannon CP, Crowe S, Woerle HJ, Broedl UC, Johansen OE (2015) Effects of empagliflozin on blood pressure and markers of arterial stiffness and vascular resistance in patients with type 2 diabetes. Diabetes Obes Metab 17:1180-1193. https://doi.org/10.1111/dom.12572

16. Sano M (2018) A new class of drugs for heart failure: SGLT2 inhibitors reduce sympathetic overactivity. J Cardiol 71:471-476.https://doi.org/10.1016/j.jjcc.2017.12.004】

17. Does dapagliflozin regress left ventricular hypertrophy in patients with type 2 diabetes? A prospective, double-blind, randomised, placebo-controlled study

18. Verma S, Garg A, Yan AT, Gupta AK, AlOmran M, Sabongui A, Teoh H, Mazer CD, Connelly KA. Effect of empagliflozin on left ventricular mass and diastolic function in individuals with diabetes:an important clue to the EMPA-REG OUTCOME trial? Diabetes Care 2016;39: e212-e213.

19. Matsutani D, Sakamoto M, Kayama Y,Takeda N, Horiuchi R, Utsunomiya K. Effect of canagliflozin on left ventricular diastolic function in patients with type 2 diabetes. Cardiovasc Diabetol 2018;17: 73.

20. Soga F, Tanaka H, Tatsumi K, Mochizuki Y, Sano H, Toki H, Matsumoto K, Shite J, Takaoka H, Doi T, Hirata KI. Impact of dapagliflozin on left ventricular diastolic function of patients with type 2 diabetic 
mellitus with chronic heart failure. Cardiovasc Diabetol 2018; 17: 132.

21. Sakai T, Miura S. Abstract 17041: effect of sodium-glucose cotransporter 2 inhibitor on vascular endothelial function and diastolic function in patients with heart failure with preserved ejection fraction (HFpEF). Circulation 2018; 136: A17041

22. Tochiya M, Makino H, Tamanaha T, Matsuo M, Hishida A, Koezuka R, Ohata Y, Tomita T, Son C, Miyamoto Y, Yasuda S, Hosoda K (2020) Effect of tofogliflozin on cardiac and vascular endothelial function in patients with type 2 diabetes and heart diseases: a pilot study. J Diabetes Invest 11:400404. https://doi.org/10.1111/jdi.13122

\section{Table}

Table 1

Overview of main characteristics of the two trial populations at baseline

\begin{tabular}{|lllll|}
\hline \multicolumn{4}{l}{ Nick S. R. Lan 2020} & \multicolumn{2}{l|}{ Subodh Verma 2019} \\
\hline & Empagliflozin & Placebo & Empagliflozin & Placebo \\
\hline Age (years) & $60.8 \pm 9.5$ & $65.1 \pm 10.4$ & 64 & 64 \\
\hline Male gender & $19(86.4)$ & $15(68.2)$ & $44(90)$ & $46(96)$ \\
\hline $\begin{array}{l}\text { Duration of T2DM, median } \\
\text { (IQR), y }\end{array}$ & $5.5(4-12.25)$ & $8(4-19.5)$ & $10.0(4.0$, & $10.0(5.0$, \\
\hline NT-pro BNP,pg/mL & $297(128-$ & $262.5(122-$ & $97(46,188)$ & $116(62,227)$ \\
\hline medications & $1497.5)$ & $806)$ & & \\
\hline ACE inhibitor & & & $40(82)$ & $41(85)$ \\
\hline
\end{tabular}

\section{Figures}




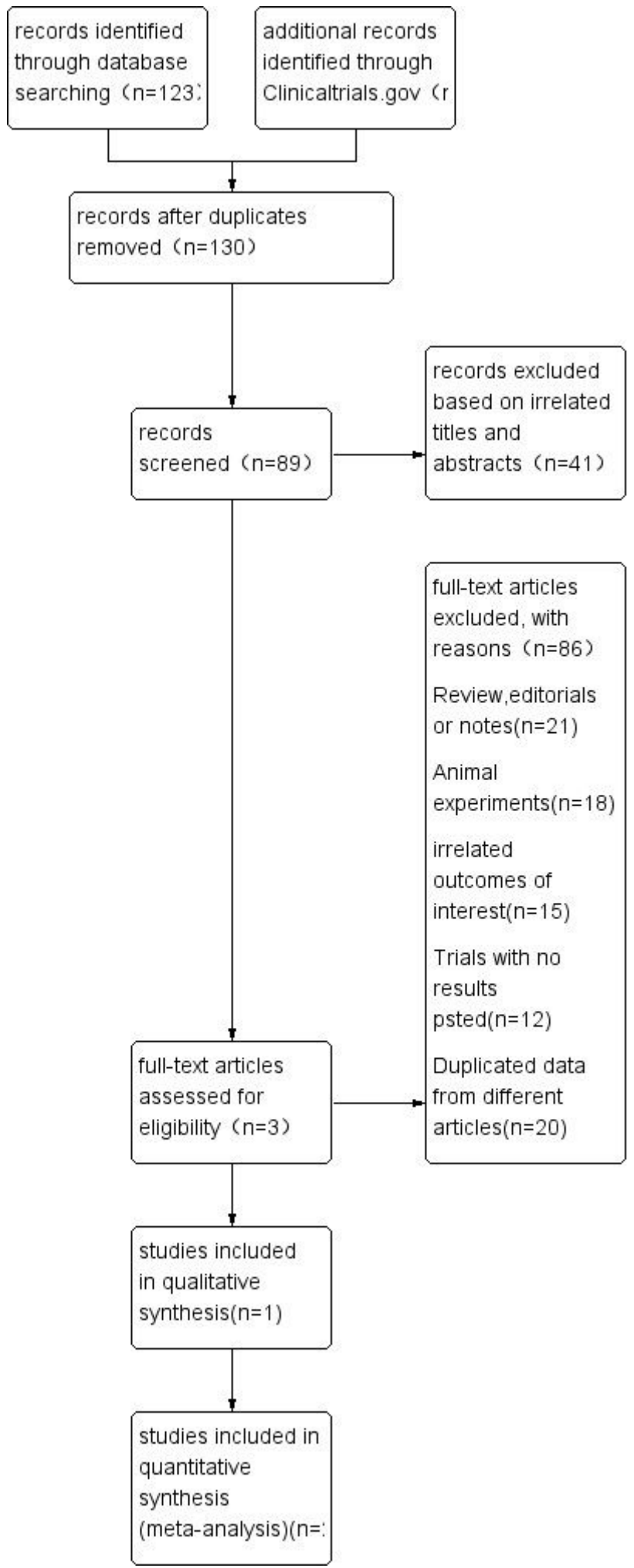

\section{Figure 1}

\section{Flow chart of literature screening}




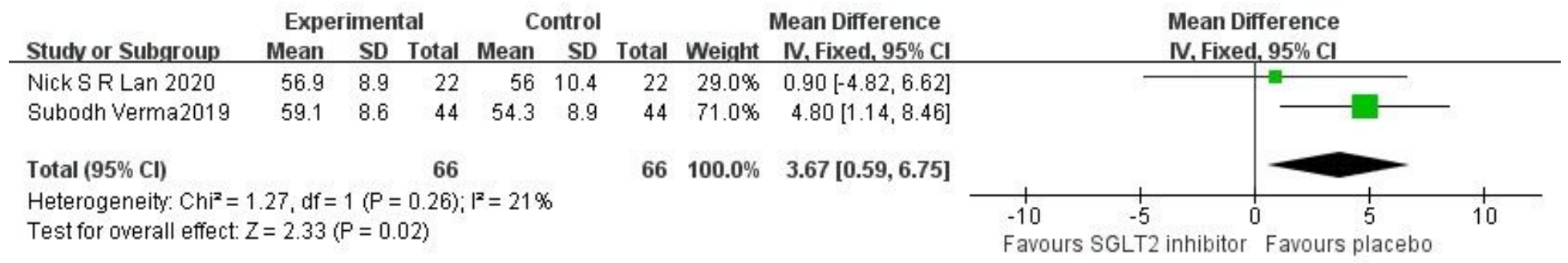

\section{Figure 2}

Forest plot of Meta-analysis of LVEF level in 2 groups after treatment

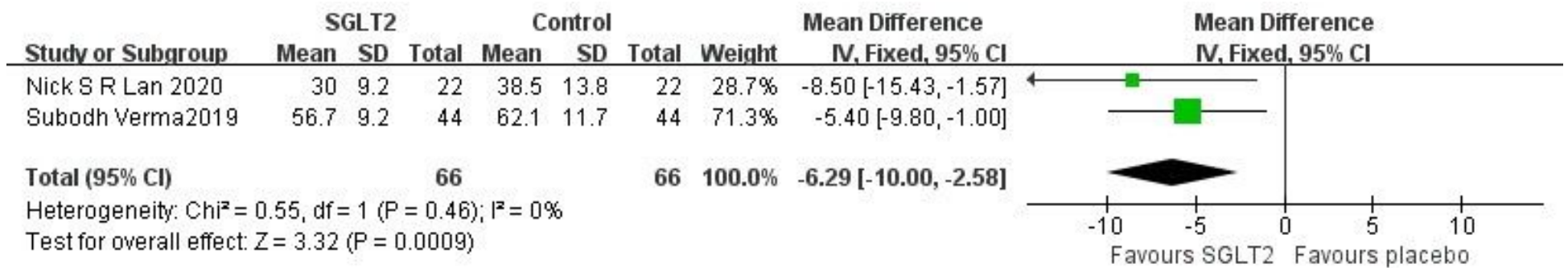

\section{Figure 3}

Forest plot of Meta-analysis of LAVI level in 2 groups after treatment

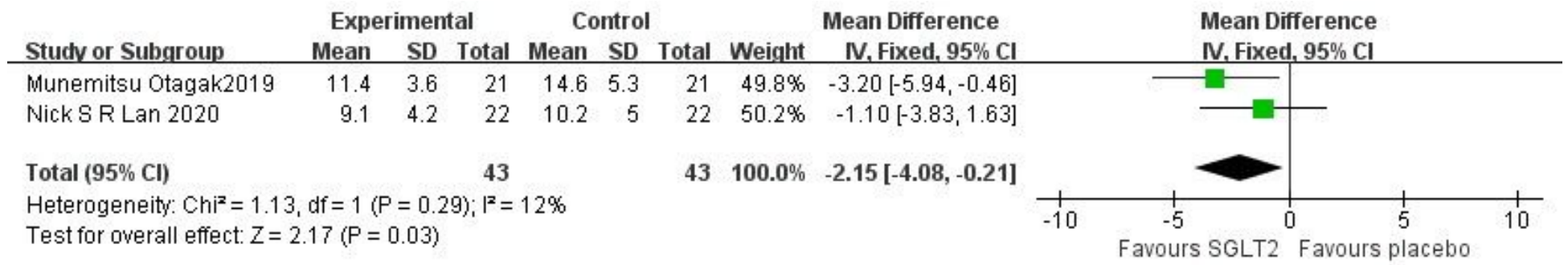

\section{Figure 4}

Forest plot of Meta-analysis of E/E' level in 2 groups after treatment

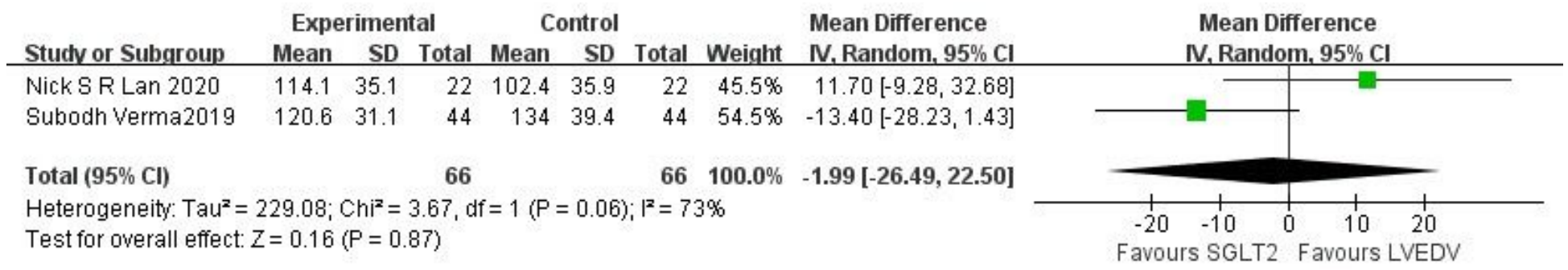

\section{Figure 5}

Forest plot of Meta-analysis of LVEDV level in 2 groups after treatment 


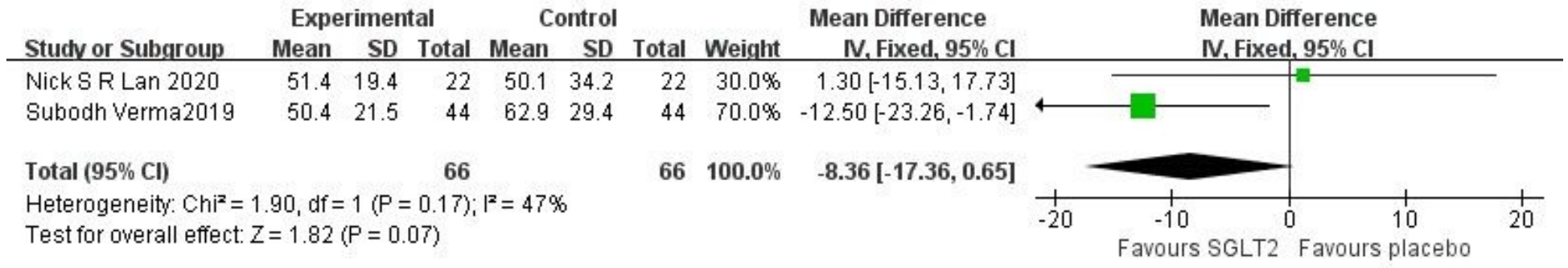

\section{Figure 6}

Forest plot of Meta-analysis of LVESV level in 2 groups after treatment 